\title{
Ten to fifteen seconds are needed for complete skin coverage with alcoholic hand disinfectant
}

\author{
Sebastian Schulz-Stübner Privatdozent Dr. med..$^{1}$, Annika Unger BA ${ }^{1}$, Michaela Berger RN ${ }^{1}$, Winfried Ebner Dr. med. ${ }^{2}$ and \\ Thomas Hauer Dr. med. ${ }^{1}$ \\ ${ }^{1}$ Deutsches Beratungszentrum für Hygiene (BZH GmbH), Freiburg, Germany and ${ }^{2}$ Institut für Infektionsprävention und Krankenhaushygiene Universitätsklinikum \\ Freiburg, Freiburg, Germany
}

To the Editor-Kramer et $\mathrm{al}^{1}$ demonstrated the microbiocidal effectiveness of 15 seconds of alcoholic hand disinfection and of reducing the time recommendations for alcoholic hand disinfection in standard operating procedures to increase compliance. However, a survey of infection control professionals in Germany revealed some concerns with implementing such a practice. $^{2}$ One such concern is the time needed to reach complete coverage of the hands with the alcoholic disinfectant. ${ }^{3}$

We conducted an experimental study during the Freiburg congress of infectious disease and infection control (Freiburger Infektiologie- und Hygienekongress) and a consulting center (BZH) meeting in 2019 to find out what volume-time combination might be sufficient to reach almost $100 \%$ skin coverage regardless of the rubbing technique used.

\section{Material and methods}

Volunteers were randomly assigned to scrub their hands as fast as they could for 5 seconds, 10 seconds, 15 seconds and 30 seconds either with $3 \mathrm{~mL}$ or $6 \mathrm{~mL}$ alcoholic hand disinfectant containing a fluorescent marker (Visirub in $500 \mathrm{~mL}$ Sterilium classic pure Paul Hartmann AG marketed by Bode Chemie Hamburg, Germany). The exact volume was placed on the palm with a prefilled syringe and the time stopped at the first counter. A video sequence of the rubbing technique was taken. A blinded observer then judged the percentage of skin coverage under ultraviolet light (Derma Lite Check Box, Bode Chemie Hamburg, Germany) and photographed the result at a second counter. Ten photos of each group were judged by a second observer as quality control. This observer also watched 10 videos with the best and worst results of each group to determine whether a specific rubbing technique giving optimal results can be identified. We used the Student $t$ test with $P<.05$ to test for significant differences between groups. Because no personal or otherwise identifying data were recorded, no written consent was needed according to the EU General Data Protection Regulation (GDPR).

\section{Results}

In total, 356 hand rubs, randomly assigned to each group, were analyzed, but 4 data sets were discarded for protocol violation. Figure 1 shows the distribution of skin coverage of each group.

Author for correspondence: Privatdozent Dr Sebastian Schulz-Stübner, E-mail: Schulz-stuebner@bzh-freiburg.de

Cite this article: Schulz-Stübner S, et al. (2020). Ten to fifteen seconds are needed for complete skin coverage with alcoholic hand disinfectant. Infection Control \& Hospital Epidemiology, 41: 488-489, https://doi.org/10.1017/ice.2019.382
The $3 \mathrm{~mL}$ per 5 seconds group was significantly worse than all other groups $(P<.001)$ and showed the largest range of coverage. A duration of 10 seconds resulted in $95 \%$ coverage regardless of volume, which was still significantly less compared to 15 and 30 seconds $(P=.0011)$. Differences between 15 and 30 seconds were not significantly different regardless of whether $3 \mathrm{~mL}$ or $6 \mathrm{~mL}$ was used $(P=.06)$. The second observer confirmed the results of the first observer, and no specific rubbing technique (classified as 3-step approach, modified 3 step-approach, 6 step-approach, modified 6-step approach and free style) could be identified as superior.

\section{Discussion}

We have shown that even within 5 seconds complete coverage of the skin of the hand is feasible by some high-performing individuals, especially if the disinfectant volume used is doubled from $3 \mathrm{~mL}$ to $6 \mathrm{~mL}$. However, the mean coverage was significantly worse and the range of coverage larger compared to longer rubbing times, independent of the volume used. Although 95\% coverage within 10 seconds seems clinically acceptable, almost complete coverage was seen after 15 seconds, with no additional benefit of longer rubbing time, independent of rubbing technique. In the Kramer study, ${ }^{1}$ the real observed disinfection time was $\sim 10$ seconds regardless of the recommended 15 seconds or 30 seconds in the standard operating procedure. Stahmeyer et $\mathrm{al}^{4}$ reported an average disinfection time of 7.6 seconds in real-life observations in intensive care units. The recommendation of 15 seconds duration for alcoholic hand disinfection seems to be safe regarding acceptable skin coverage. Microbiocidal effectiveness has been proven elsewhere. ${ }^{1,5}$ In addition to the benefit of increasing overall compliance, a realistic time recommendation might also help increase the real rubbing time, which would result in more optimal skin coverage. Clearly, best performers exist among the hand rubbers. Multiple different techniques were used and no unique technique could be identified as superior. This finding is consistent with the results of Tschudin-Suter et al, who described the similar effectiveness of a 3-step approach compared to the conventional 6-step approach under experimental conditions ${ }^{6}$ and in real life. ${ }^{7}$ Our study was limited by the fact that the participants were infection control practitioners and physicians with special interest in hospital hygiene. However, these groups of healthcare workers might be highly theoretically trained but maybe not the most frequent performers in daily life. Thus, we believe that our results can be extrapolated to healthcare workers in general and that optimal skin coverage with an alcoholic hand disinfectant can be reached within $10-15$ seconds. 
Fig. 1. Mean skin coverage in $\%$ and range of distribution with different volume/time combinations using fluorescent marker in alcoholic hand disinfectant.

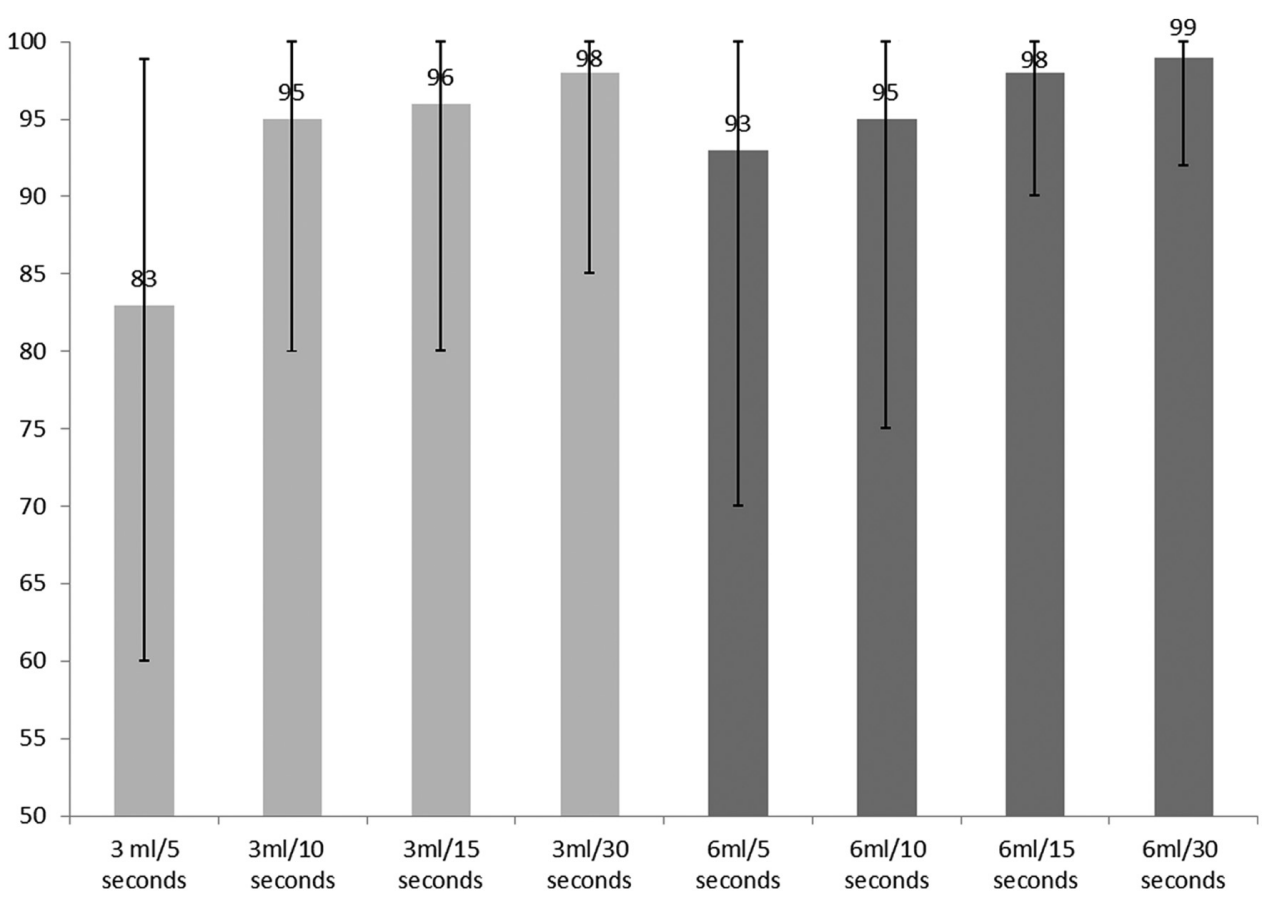

Acknowledgments. None.

Financial support. This work was funded by institutional funds only.

Conflicts of interest. S.S.S. is a shareholder of BZH-Beteiligungs-GbR and receives royalties from Springer, Thieme, and Consilium infectiorum. T.H. is a shareholder of BZH-Beteiligungs-GbR and receives royalties from Springer, Elsevier, and Thieme.

\section{References}

1. Kramer A, Pittet D, Klasinc R, Krebs S, Koburger T, Fusch C, Assadian O. Shortening the application time of alcohol-based hand rubs to 15 seconds may improve the frequency of hand antisepsis actions in a neonatal intensive care unit. Infect Control Hosp Epidemiol 2017;38:1430-1434.

2. Schulz-Stübner S, Volkmann A, Ebner W, Hauer T. Practice and attitudes toward alcohol based hand disinfection among German infection control teams. Infect Control Hosp Epidemiol 2019;40:609-612.

3. Deutsche Gesellschaft für Krankenhaushygiene (DGKH)/German Society of Hospital hygiene. Stellungnahme des Vorstandes der DGKH zur
Verkürzung der Einwirkzeit der Händedesinfektion von 30 aus 15 Sekunden [Statement of the DGKH board in regard to shortening the recommendation for hand disinfection from 30 to 15 seconds. Hyg Med 2019;44:190-191.

4. Stahmeyer JT, Lutze B, von Lengerke T, Chaberny IF, Krauth C, Hand Hygiene in intensive care units: a matter of time? J Hosp Infect 2017;95: 338-343.

5. Pires D, Spule H, Bellissimo-Rodrigues F, Gayet-Ageron A, Pittet D. Hand hygiene with alcohol-based hand rub: how long is long enough? Infect Control Hosp Epidemiol 2017;38:547-552.

6. Tschudin-Suter S, Rotter ML, Frei R, et al. Simplifying the WHO 'how to hand rub' technique: three steps are as effective as six-results from an experimental randomized crossover trial. Clin Microbiol Infect 2017;23: 409.e1-409.e4.

7. Tschudin-Sutter S, Sepulcri D, Dangel M, Ulrich A, Frei R, Widmer AF. Simplifying the WHO protocol: three steps versus six steps for performance of hand hygiene-a cluster-randomized trial. Clin Infect Dis 2018 Nov 3. doi: $10.1093 /$ cid/ciy 948 .

\section{The varying specificity of urine cultures in different populations}

\section{Kirk M. Chan-Tack MD ${ }^{1,2}$ (1), Barbara W. Trautner MD, PhD ${ }^{3,4}$ and Daniel J. Morgan MD, MS ${ }^{1,5}$}

${ }^{1}$ Veterans' Affairs Maryland Health Care System, Baltimore, Maryland, ${ }^{2}$ Center for Drug Evaluation and Research, Food and Drug Administration, Silver Spring, Maryland, ${ }^{3}$ Center for Innovations in Quality, Effectiveness, and Safety at the Michael E. DeBakey Veterans' Affairs Medical Center, Houston, Texas, ${ }^{4}$ Department of Medicine, Baylor College of Medicine, Houston, Texas and ${ }^{5}$ Division of Genomic Epidemiology and Clinical Outcomes, Department of Epidemiology and Public Health, Baltimore, Maryland

\footnotetext{
Health,

Author for correspondence: Daniel J. Morgan MD, MS, Epidemiology and Public

University of Maryland School of Medicine, 10 S Pine St, Medical School Teaching Facility 334, Baltimore, MD 21211. E-mail: dmorgan@som. umaryland.edu

Cite this article: Chan-Tack KM, Trautner BW, and Morgan DJ. (2020). The varying specificity of urine cultures in different populations. Infection Control \& Hospital Epidemiology, 41: 489-491, https://doi.org/10.1017/ice.2020.16
}

To the Editor-Diagnostic testing is essential in distinguishing patients who have a disease from those who do not. The accuracy of a test is described by sensitivity and specificity. Sensitivity reflects how many patients with disease have a positive test, and specificity reflects how many patients without disease have a 